\title{
Article
}

\section{Risk Factors Associated with 30-Day Mortality in Older Patients with Influenza}

\author{
Charles Guesneau ${ }^{1}$, Anne Sophie Boureau ${ }^{1}$ () , Céline Bourigault ${ }^{2,3}$, Gilles Berrut ${ }^{1}$, Didier Lepelletier ${ }^{2,3}$, \\ Laure de Decker ${ }^{1,2}$ and Guillaume Chapelet ${ }^{1,2, *}$
}

1 Clinical Gerontology Department, Nantes University Hospital, 1 Place Alexis-Ricordeau, F-44000 Nantes, France; charles.guesneau@chu-nantes.fr (C.G.); annesophie.boureau@chu-nantes.fr (A.S.B.); gilles.berrut@chu-nantes.fr (G.B.); laure.dedecker@chu-nantes.fr (L.d.D.)

2 Université de Nantes, EE MiHAR (Microbiotes, Hôtes, Antibiotiques et Résistance Bacterienne), Institut de Recherche en Santé (IRS2), 22 Boulevard Bénoni-Goullin, F-44200 Nantes, France; celine.bourigault@chu-nantes.fr (C.B.); didier.lepelletier@chu-nantes.fr (D.L.)

3 Bacteriology and Infection Control Department, Nantes University Hospital, 1 Place Alexis-Ricordeau, F-44000 Nantes, France

* Correspondence: guillaume.chapelet@chu-nantes.fr

check for updates

Citation: Guesneau, C.; Boureau, A.S.; Bourigault, C.; Berrut, G.; Lepelletier, D.; de Decker, L.; Chapelet, G. Risk Factors Associated with 30-Day Mortality in Older Patients with Influenza. J. Clin. Med. 2021, 10, 3521. https://doi.org/ $10.3390 /$ jcm 10163521

Academic Editors: Patrick Manckoundia and Alain Putot

Received: 12 July 2021

Accepted: 2 August 2021

Published: 11 August 2021

Publisher's Note: MDPI stays neutral with regard to jurisdictional claims in published maps and institutional affiliations.

Copyright: (c) 2021 by the authors. Licensee MDPI, Basel, Switzerland. This article is an open access article distributed under the terms and conditions of the Creative Commons Attribution (CC BY) license (https:// creativecommons.org/licenses/by/ $4.0 /)$.

\begin{abstract}
Background: Influenza is a common viral condition, but factors related to short-term mortality have not been fully studied in older adults. Our objective was to determine whether there is an association between geriatric factors and 30-day mortality. Methods: This was a retrospective cohort design. All patients aged 75 years and over, with a diagnosis of influenza confirmed by a positive RT-PCR, were included. The primary endpoint was death within the 30 days after diagnosis. Results: 114 patients were included; 14 (12.3\%) patients died within 30 days. In multivariate analysis these patients were older (OR: 1.37 95\% CI $(1.05,1.79), p=0.021)$, and had a lower ADL score (OR: 0.36 95\% CI $(0,17 ; 0.75), p=0.006)$, and a higher SOFA score (OR: $2.3095 \%$ CI $(1.07,4.94)$, $p=0.03$ ). Oseltamivir treatment, initiated within the first $48 \mathrm{~h}$, was independently associated with survival (OR: $0.0495 \%$ CI $(0.002,0.78), p=0.034)$. Conclusions: Identification of mortality risk factors makes it possible to consider specific secondary prevention measures such as the rapid introduction of antiviral treatment. Combined with primary prevention, these measures could help to limit the mortality associated with influenza in older patients.
\end{abstract}

Keywords: influenza; elderly; risk factors; mortality

\section{Introduction}

It has been estimated that the global prevalence of influenza is around 1 billion cases annually. Of these, 3-5 million cases are severe, causing between 290,000 and 650,000 deaths (i.e., 4.0-8.8 per 100,000 individuals) [1], especially among people aged 75 years or older (51.3-99.4 per 100,000 individuals) [1]. In France, the incidence of deaths exceeds 9000 per season, corresponding to $11 \%$ of all-cause deaths during the epidemic season in the entire population [2].

Mortality is higher in elderly patients, and influenza-related deaths increase exponentially after the age of 65 [3]. However, few studies have highlighted the impact of specific geriatric criteria on short-term mortality. Several studies have identified variables associated with influenza-related mortality [4-14], but very few have focused specifically on elderly patients [15-17]. In these studies, the following factors were associated with shortterm mortality: age [6,12]; presence of underlying diseases [13] including diabetes [5,8] immunosuppression $[4,7,8,17]$, chronic respiratory disease [6,7], chronic cardiac disease [6,14], renal disease [6], and cancer [7,12]; number of drugs being administered [6,16]; malnutrition [16]; recent infectious disease [16]; severity of illness [4,5,10,12,14,17] including bacterial co-infection [9] and nosocomial infection [9]; delays in adequate treatment and 
admission to the intensive care unit $[7,8,11]$; delays in the initiation of antiviral treatment (within the first 48 h) $[8,9,13,17]$; and number of consultations in the past year [15].

Our hypothesis was that geriatric factors are associated with short-term mortality in elderly patients with seasonal influenza. We analyzed data during the epidemic period between January and March 2015, when an increased prevalence of influenza was described by the European Influenza Surveillance Scheme (EISS) [18]. Our objective was to determine whether there was an association between geriatric variables and 30-day mortality in patients with seasonal influenza.

\section{Materials and Methods}

\subsection{Study Population}

This retrospective cohort study was conducted in 2017 in a tertiary, 2600-bed, universityaffiliated center at the Clinical Gerontology Department, Nantes, France. The Clinical Gerontology Department is composed of 2 acute-care services, 2 post-acute care services, and 2 nursing home residences.

From January to April 2015, all patients who were 75 years of age or older were retrospectively included in this study if they met all the following criteria: (i) influenza-like symptoms (fever and/or respiratory signs and/or myalgias and/or headache); (ii) positive RT-PCR (detection of influenza A or B); and (iii) no admission to ICU. For each patient included, only the first episode of positive RT-PCR was considered. Patients who did not meet the detailed definition of the inclusion criteria were not included.

\subsection{Data Collection}

Our primary endpoint was presence/absence of death within the 30 days after the diagnosis of influenza to investigate factors associated with short-term mortality. The analyses of medical records and 2 death notification registers (www.avis-de-deces.net; www.dansnoscoeurs.fr) were considered to assess the primary outcome. General practitioners were called if the outcome was not available in the medical record and the death notification registers.

Considering the results of the previous studies focused on short-term mortality in general population [4-14] and in elderly patients [15-17], the following information was collected concerning patient's characteristics: age; sex; presence of an underlying disease including diabetes, heart disease, chronic lung disease, chronic obstructive pulmonary disease (COPD), renal disease, cancer, and hypogammaglobulinemia; the Charlson's Comorbidity Index score [19]; the Katz Index of Independence in Activities of Daily Living (ADL) [20]; the number of drugs being administered including statins/immunosuppressive/corticoids; and influenza vaccination status. Moreover, concerning influenza infection the following variables were collected: detection of influenza A or B viruses by RT-PCR; date of sampling and delay (in days) between diagnosis and the onset of symptoms; hospitalization or admission to an intensive care unit; nosocomial influenza infection (infection acquired at least after $48 \mathrm{~h}$ of hospitalization in the acute care services or in the post-acute care service); severity of infection i.e., SOFA score at the diagnosis [21]; prescription of antiviral treatment, including the delay between the onset of symptoms and antiviral prescription; and antibiotic co-prescription.

Considering previous studies that had highlighted factors associated with short-term mortality, "chronic respiratory disease" corresponds to patients with history of chronic lung disease and/or chronic obstructive pulmonary disease (COPD), and "immunosuppression" corresponds to patients with active cancer and/or hemopathy, or history of cancer and/or hemopathy in the last 5 years, and/or hypogammaglobulinemia, and/or treatment with anti-inflammatory and/or immunosuppressive drugs, including corticosteroids.

\subsection{Statistical Analysis}

The participant's baseline characteristics were summarized using mean and standard deviations or frequencies and percentages, as appropriate. The analyzed variable of interest 
was short-term mortality, i.e., mortality in the 30 days following the diagnosis of infection. Between-group comparisons were performing using an independent simple t-test or chisquared test (X2), as appropriate. The Shapiro-Wilk test was used to assess the normality of continuous variables. Non-normally distributed continuous variables were compared using the Mann-Whitney test. Normally distributed continuous variables were compared using Student's t-test. Univariable and multivariable logistic regressions were performed to examine the association between short-term mortality and other variables. Variables with a significant association in univariate analysis and/or with a $p$-value $<0.2$ were entered into a logistic regression model for multivariate analysis. Relative risks were expressed as odds ratios (OR) and 95\% confidence intervals. All reported $p$-values $<0.05$ were considered as statistically significant. Analysis was performed using SPSS software version 15.0 (SPSS, Inc., Chicago, IL, USA).

\section{Results}

\subsection{Patient Characteristics}

From January to March 2015, 122 nasopharyngeal swabs tested positive for the detection of the influenza virus by RT-PCR at the Clinical Gerontology Department (Figure 1): $115(94.3 \%)$ were positive for influenza A, and $7(5.7 \%)$ for influenza B. One patient had two positive RT-PCR results. For this patient, we considered only the first episode (influenza $B$ detection by RT-PCR), but not the second episode (influenza A detection by RT-PCR). Four patients had positive RT-PCR results but without influenza-like symptoms. Three patients were aged under 75 years old. Finally, 114 patients were included. Positive RT-PCR distributions over time are detailed in Supplementary Figure S1. The repartition of positive results according to the different units of the Clinical Gerontology Department are detailed in Supplementary Table S1. Patient characteristics $(n=114)$ are detailed in Table $1 ; 82$ $(71.9 \%)$ were female, median age $( \pm \mathrm{SD}) 87.9 \pm 5.5$. The mean Charlson Comorbidity Index score $( \pm \mathrm{SD})$ was $2.83( \pm 1.7)$, and the mean ADL score $( \pm \mathrm{SD})$ was $3.08( \pm 2.1)$.

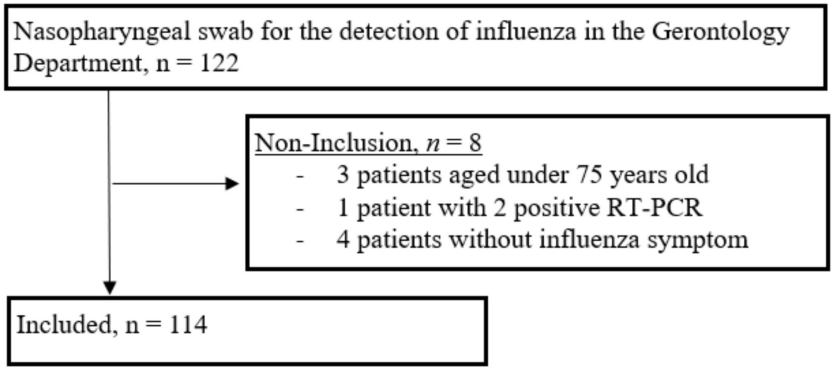

Figure 1. Screening, exclusion, and enrollment process of participants.

Table 1. Demographic and clinical characteristics of the total population, $n=114$.

\begin{tabular}{|c|c|c|c|c|c|}
\hline & $\begin{array}{c}\text { Total } \\
n=114\end{array}$ & $\begin{array}{c}\text { Survivors } \\
n=100(87.7 \%)\end{array}$ & $\begin{array}{c}\text { Non-Survivors } \\
\begin{array}{c}n=14 \\
(12.3 \%)\end{array}\end{array}$ & Missing Data & $p$-Value \\
\hline \multicolumn{6}{|l|}{ Patient characteristics } \\
\hline Age, years, mean, \pm SD & $87.9 \pm 5.5$ & $87.3 \pm 5.4$ & $91.5 \pm 4.7$ & - & 0.006 \\
\hline Gender, female, $n(\%)$ & $82(71.9)$ & $72(72)$ & $10(71.4)$ & - & 1.000 \\
\hline $\begin{array}{l}\text { Charlson Comorbidity Index } \\
\text { score }, \pm \mathrm{SD}\end{array}$ & $2.83 \pm 1.7$ & $2.73 \pm 1.7$ & $3.57 \pm 1.7$ & - & 0.119 \\
\hline Diabetes, $n(\%)$ & $25(21.9)$ & $23(23)$ & $2(14.3)$ & - & 0.731 \\
\hline Chronic renal disease, $n(\%)$ & $15(13.2)$ & $14(14)$ & $1(7.1)$ & - & 0.690 \\
\hline Chronic respiratory disease, $n(\%)$ & $16(14)$ & $13(13)$ & $3(21.4)$ & - & 0.413 \\
\hline Chronic cardiac disease, $n(\%)$ & $37(32.5)$ & $29(29)$ & $8(57.1)$ & - & 0.063 \\
\hline Immunosuppression, $n(\%)$ & $17(14.9)$ & $15(15)$ & $2(14.3)$ & - & 1.000 \\
\hline $\mathrm{ADL}$, mean $\pm \mathrm{SD}$ & $3.08 \pm 2.1$ & $3.25 \pm 2.1$ & $1.82 \pm 1.7$ & - & 0.020 \\
\hline
\end{tabular}


Table 1. Cont.

\begin{tabular}{|c|c|c|c|c|c|}
\hline & $\begin{array}{c}\text { Total } \\
n=114\end{array}$ & $\begin{array}{c}\text { Survivors } \\
n=100(87.7 \%)\end{array}$ & $\begin{array}{c}\text { Non-Survivors } \\
n=14 \\
(12.3 \%)\end{array}$ & Missing Data & $p$-Value \\
\hline Number of drugs, $n \pm \mathrm{SD}$ & $6.32 \pm 3.0$ & $6.15 \pm 3.2$ & $7.50 \pm 1.8$ & - & 0.026 \\
\hline Statin therapy, $n(\%)$ & $23(20.2)$ & $20(20)$ & $3(21.4)$ & - & 1.000 \\
\hline Vaccination, $n(\%)$ & $44(38.6)$ & $37(37)$ & $7(50)$ & $70(61.4)$ & 0.576 \\
\hline \multicolumn{6}{|l|}{ Influenza infection characteristics } \\
\hline Influenza $\mathrm{A}, n(\%)$ & $107(93.9)$ & $93(93)$ & $14(100)$ & - & 0.594 \\
\hline $\begin{array}{l}\text { Delay between diagnosis and the } \\
\text { onset of symptoms, days, } n \pm \mathrm{SD}\end{array}$ & $2.91 \pm 3.5$ & $2.94 \pm 3.6$ & $2.71 \pm 2.1$ & - & 0.408 \\
\hline Nosocomial infection, $n(\%)$ & $35(30.7)$ & $32(32)$ & $3(21.4)$ & - & 0.545 \\
\hline Antiviral $<48 \mathrm{~h}, n(\%)$ & $48(42.1)$ & $45(45)$ & $3(21.4)$ & - & 0.147 \\
\hline Pneumonia, $n(\%)$ & $56(49.1)$ & $46(46)$ & $10(71.4)$ & - & 0.092 \\
\hline Antibiotic prescription, $n(\%)$ & $61(53.5)$ & $50(50)$ & $11(78.6)$ & - & 0.051 \\
\hline SOFA score, mean \pm SD & $1.3(1,4)$ & $1.12(1.3)$ & $2.57(1.6)$ & - & 0.001 \\
\hline Lymphopenia, $n(\%)$ & $77(73.3)$ & $66(66)$ & $11(78.6)$ & $9(7.9)$ & 0.506 \\
\hline
\end{tabular}

SD, Standard Deviation; ADL, Activities of Daily Living.

\subsection{Factors Associated with Short-Term Mortality}

The 30-day mortality incidence was $12.3 \%$ (14/114). The characteristics of survivors and non-survivors are shown in Table 1 . The comparison between groups revealed significant differences concerning the following factors: age $(p=0.006)$; mean ADL score $(p=0.020)$; mean number of drugs $(p=0.026)$; and mean SOFA score $(p=0.001)$. No differences between groups were observed in terms of morbidity, including the Charlson Comorbidity Index and other influenza infection characteristics.

In univariate analysis (Table 2), the three following factors were significantly associated with short-term mortality: age $(\mathrm{OR}=1.18,95 \% \mathrm{CI}=(1.04-1.35), p=0.013)$; ADL score $(\mathrm{OR}=0.69,95 \% \mathrm{CI}=(0.05-0.95), p=0.027)$; and SOFA score $(\mathrm{OR}=1.83,95 \% \mathrm{CI}=(1.27-2.64)$, $p=0.001)$.

Table 2. Univariate and multivariate logistic regression analysis of factors associated with mortality.

\begin{tabular}{ccccc}
\hline & \multicolumn{2}{c}{ Univariate Analysis } & \multicolumn{2}{c}{ Multivariate Analysis } \\
& OR (95\% CI) & $p$-Value & OR (95\% CI) & 0.021 \\
Age & $1.18(1.04 ; 1.35)$ & 0.013 & $1.37(1.05 ; 1.79)$ & 0.342 \\
Female & $0.97(0.28 ; 3.36)$ & 0.964 & $3.01(0.31 ; 29.20)$ & 0.268 \\
Charlson Comorbidity Index & $1.31(0.96 ; 1.80)$ & 0.092 & $1.39(0.78 ; 2.48)$ & 0.662 \\
Diabetes & $0.56(0.12 ; 2.68)$ & 0,466 & $0.60(0.06 ; 5.91)$ & 0.334 \\
Chronic respiratory disease & $1.83(0.45 ; 7.43)$ & 0.401 & $0.27(0.02 ; 3.91)$ & 0.134 \\
Chronic cardiac disease & $3.26(1.04 ; 10.24)$ & 0.043 & $6.48(0.56 ; 74.69)$ & 0.683 \\
Immunosuppression & $0.94(0.19 ; 4.65)$ & 0.944 & $1.62(0.16 ; 16.40)$ & 0.006 \\
ADL score & $0.69(0.50 ; 0.95)$ & 0.027 & $0.36(0.17 ; 0.75)$ & 0.405 \\
Number of drugs & $1.16(0.96 ; 1.40)$ & 0.128 & $1.15(0.83 ; 1.61)$ & 0.545 \\
Nosocomial infection & $0.58(0.15 ; 2.22)$ & 0.426 & $2.17(0.18 ; 26.76)$ & 0.034 \\
Antiviral <48 h & $0.33(0.09 ; 1.27)$ & 0.107 & $0.04(0.002 ; 0.78)$ & 0.704 \\
Antibiotic prescription & $3.67(0.97 ; 13.94)$ & 0.057 & $0.64(0.07 ; 6.28)$ & 0.034 \\
SOFA score & $1.83(1.27 ; 2.64)$ & 0.001 & $2.30(1.07 ; 4.94)$ & 0.453 \\
Lymphopenia & $2.17(0.45 ; 10.45)$ & 0.336 & $0.42(0.04 ; 4.03)$ & \\
\hline
\end{tabular}

OR, Odds Ratios; ADL, Activities of Daily Living; SOFA, Sequential Organ Failure Assessment .

In the multivariate analysis (Table 2), the four following factors were significantly associated with short-term mortality: age $(\mathrm{OR}=1.37,95 \% \mathrm{CI}=(1.05-1.79), p=0.021)$; ADL score $(\mathrm{OR}=0.36,95 \% \mathrm{CI}=(0.17-0.75), p=0.006)$; antiviral treatment administration in the first $48 \mathrm{~h}(\mathrm{OR}=0.04,95 \% \mathrm{CI}=(0.002-0.78), p=0.034)$; and SOFA score $(\mathrm{OR}=2.30$, $95 \% \mathrm{CI}=(1.07-4.94), p=0.034)$. 


\section{Discussion}

Influenza is a common viral condition that can be serious and even fatal in some at-risk populations, including that of older adults. Indeed, mortality is higher in older adults, and influenza-related death increases exponentially after the age of 65 [3]. Few studies have highlighted the impact of specific geriatric factors on short-term mortality. In this study an elevated ADL score and antiviral treatment administration within the first $48 \mathrm{~h}$ of symptoms were associated with reduced 30-day mortality, while a high SOFA score and age were associated with increased 30-day mortality.

In the multivariable analysis, age was significantly associated with increased shortterm mortality during seasonal influenza epidemics in elderly patients. This result is consistent with previous clinical and epidemiologic studies $[1,2,6,12,13]$ and was confirmed by Mertz et al. in a recent meta-analysis [22]. However, we assume that it could be difficult to distinguish physiological age from chronological age [23], and this result could be biased by confounding factors such as the immunological markers of immunosenescence [24].

In this study, a higher ADL score [20], i.e., a higher ability to performed activities daily living, is associated with a reduced short-term mortality. This result is consistent with the study of Gozalo et al. [16], which highlighted the relationship between influenza and a decline in activities of daily living in nursing home $(\mathrm{NH})$ residents.

In this study, a higher SOFA score was associated with short-term mortality. This result is not surprising because this association was described in a previous study that included ICU patients [12]. Our study confirms this association in an elderly population. Previous studies also found an association between short-term influenza mortality and other severity scores such as the SAPS II score [14] and APACHE score [17]. In this study, we used the SOFA score because its use is consensual in assessing the severity of sepsis [21].

In our study, antiviral (oseltamivir) prescription within the first $48 \mathrm{~h}$ was associated with reduced short-term mortality. It was the only modifiable variable associated with mortality in our studies, even if this association could be due to the above and other potential biases and confounders. Although the impact on mortality of antiviral prescription is controversial [25], this result is consistent with previous studies $[8,9,13,17]$. Antiviral treatment can be prescribed in the elderly population, as was proposed by the Centers for Disease Control and Prevention (CDC) Advisory Committee on Immunization Practices (ACIP) in the two following recommendations [26]: (i) early antiviral treatment prescription is proposed for patients with suspected or confirmed severe influenza (e.g., those who have severe, complicated, or progressive illness or who require hospitalization), (ii) early antiviral treatment prescription is proposed for patients with of suspected or confirmed influenza at higher risk for influenza complications.

In this study, we could not analyze the association between history of influenza vaccination and short-term mortality because of missing data $(n=70(61.4 \%))$. Although influenza vaccination had a positive but modest impact in preventing influenza, its impact on the reduction of mortality is controversial, especially in older patients. The 2010 Cochrane meta-analysis did not conclude that influenza vaccination is effective in people aged 65 and over [27]. However, the critical analysis of this meta-analysis highlighted a vaccine efficacy of $30 \%$ for the prevention of lethal and non-lethal influenza complications, $40 \%$ for the prevention of clinical influenza, $50 \%$ for virologically confirmed influenza, and $60 \%$ for the prevention of biological influenza infection [28]. Another study from Fireman et al. [29] highlighted a vaccine effectiveness against all-cause mortality of $4.6 \%$ (0.7-8.3), ranging from $5.3 \%$ in people aged 65 to 79 to $3.9 \%$ in the oldest age group. This effectiveness was estimated at $40 \%$ for the prevention of influenza-related deaths. Another meta-analysis conducted in 2018 [27] showed a lower risk of contracting influenza $(6-2.4 \%)$ and influenza syndrome $(6-3.5 \%)$ in vaccinated elderly patients. However, the hospitalization and mortality data were insufficient. Finally, influenza vaccination has a significant effectiveness in preventing influenza-related deaths and reducing the risk of hospitalization, but a moderate effectiveness in preventing deaths from all causes. Society should invest in research on a new generation of influenza vaccines for the elderly [27]. 
We did not observe an association between short-term mortality and the following factors that were previously associated with short-term mortality in patients with influenza: presence of underlying diseases [13] including diabetes [5,8], immunosuppression [4,7,8,17], chronic respiratory disease [6,7], chronic cardiac disease [6,14], renal disease [6], and cancer [7,12]; number of drugs being received [6,16]; malnutrition [16]; recent infectious disease [16] including bacterial co-infection [9]; nosocomial infection [9]; and number of consultations in the past year [15]. Three main methodological biases could explain these results. First, our study may have been underpowered to detect associations. Specifically, nutritional status and oral hygiene could be important factor associated with mortality, and exploration of this aspect was limited due to missing data associated with the retrospective design. Second, in previous studies all confounding factors were not included in the multivariate analysis. Third, our population could be different with regard to the presence and the number of underlying diseases. We hypothesized that this heterogeneity of underlying disease could have biased our results. However, we did not observe an association between mortality and the Charlson comorbidity index score. Finally, we hypothesized that a global score of comorbidities may have less impact than specific comorbidities in short-term mortality, and/or that our study may have been underpowered to detect this.

This study has several limitations. First, this study had an observational design, and we reported the results of a retrospective analysis with a small number of cases, controls, and events (14 deaths). Therefore, this study may have been underpowered for the definitive validation of these results. Hidden bias may exist, with the underestimation of the true relationship between some factors (such as environmental or social factors) and short-term mortality. Second, this was a monocentric study, and the results may not be fully applicable to other settings. However, we assume that our population is representative, and we observed 30-day all-cause mortality. The mortality incidence of $12.3 \%$ is consistent with that observed in Europe during this period [18]. Third, all geriatric conditions (such as frailty) could not be included because of the retrospective study design. These factors should be analyzed in further studies. Fourth, risk factors found in one season may reflect an epidemic and not the usual year. Prospective studies should be performed considering multiple years. Fifth, confounding bias by indication could alter the association between oseltamivir treatment and mortality, because the allocation of treatment was not randomized. Sixth, our studies found only one modifiable variable associated with mortality (oseltamivir treatment). However, there is still benefit in quantifying nonmodifiable risks for the benefit of prognosis and monitoring.

\section{Conclusions}

This study among elderly patients with influenza highlights that antiviral treatment administration within the first $48 \mathrm{~h}$ of symptoms may reduce 30-day mortality, while a high SOFA score and age may increase it. Further studies are necessary to confirm our results and analyze the impact of geriatric conditions, malnutrition, functional or frailty status, and environmental or social factors on short-term mortality in patients with influenzae. These results could help to develop strategies in order to optimize preventive and curative management of influenza in elderly patient.

Supplementary Materials: The following are available online at https:/ / www.mdpi.com/article/10 $.3390 /$ jcm10163521/s1, Supplementary Figure S1: Distribution over time of the number of positive influenza RT-PCR, $(n=122)$, Supplementary Table S1: Screening, non-inclusion, and enrollment process of participants according to the site of inclusion (Clinical Gerontology Department).

Author Contributions: Concept and design, A.S.B., G.C., L.d.D.; acquisition, analysis, and interpretation of data, A.S.B., C.B., G.C., L.d.D., C.G.; drafting and revising the article critically for important intellectual content, G.C., L.d.D., G.B., D.L. All authors have read and agreed to the published version of the manuscript.

Funding: This research received no external funding. 
Institutional Review Board Statement: The study was conducted in accordance with the ethical standards set forth in the Helsinki declaration (1983). This study was approved by the local ethics committee of the Centre Hospitalier Universitaire of Nantes, and the Groupe Nantais d'Ethique dans le Domaine de la Santé (GNEDS). The identifying information of each patient was encrypted in an electronic database.

Informed Consent Statement: Patient consent was waived due to the retrospective design, in accordance with the institutional review board statement.

Data Availability Statement: Data available on request due to restrictions eg privacy or ethical.

Conflicts of Interest: The authors declare no conflict of interest.

\section{References}

1. Iuliano, A.D.; Roguski, K.M.; Chang, H.H.; Muscatello, D.J.; Palekar, R.; Tempia, S.; Cohen, C.; Gran, J.M.; Schanzer, D.; Cowling, B.J.; et al. Estimates of global seasonal influenza-associated respiratory mortality: A modelling study. Lancet 2018, 391, 1285-1300. [CrossRef]

2. Bonmarin, I.; Belchior, E.; Lévy-Bruhl, D. Impact of influenza vaccination on mortality in the French elderly population during the 2000-2009 period. Vaccine 2015, 33, 1099-1101. [CrossRef]

3. Pop-Vicas, A.; Gravenstein, S. Influenza in the elderly: A mini-review. Gerontology. 2011, 57, 397-404. [CrossRef]

4. Li, G.; Yilmaz, M.; Kojicic, M.; Fernández-Pérez, E.; Wahab, R.; Huskins, W.C.; Afessa, B.; Truwit, J.D.; Gajic, O. Outcome of critically ill patients with influenza virus infection. J. Clin. Virol. 2009, 46, 275-278. [CrossRef] [PubMed]

5. Xi, X.; Xu, Y.; Jiang, L.; Li, A.; Duan, J.; Du, B.; Chinese Critical Care Clinical Trial Group (2010). Hospitalized adult patients with 2009 influenza A(H1N1) in Beijing, China: Risk factors for hospital mortality. BMC Infect. Dis. 2010, 10, 256. [CrossRef]

6. Vandermeer, M.L.; Thomas, A.R.; Kamimoto, L.; Reingold, A.; Gershman, K.; Meek, J.; Farley, M.M.; Ryan, P.; Lynfield, R.; Baumbach, J.; et al. Association between use of statins and mortality among patients hospitalized with laboratory-confirmed influenza virus infections: A multistate study. J. Infect. Dis. 2012, 205, 13-19. [CrossRef]

7. Chowell, G.; Ayala, A.; Berisha, V.; Viboud, C.; Schumacher, M. Risk factors for mortality among 2009 A/H1N1 influenza hospitalizations in Maricopa County, Arizona, April 2009 to March 2010. Comput. Math. Methods Med. 2012, 2012, 914196. [CrossRef] [PubMed]

8. Balaganesakumar, S.R.; Murhekar, M.V.; Swamy, K.K.; Kumar, M.R.; Manickam, P.; Pandian, P. Risk factors associated with death among influenza A (H1N1) patients, Tamil Nadu, India, 2010. J. Postgrad. Med. 2013, 59, 9-14.

9. Ergönül, Ö.; Alan, S.; Ak, Ö.; Sargın, F.; Kantürk, A.; Gündüz, A.; Engin, D.; Öncül, O.; Balkan, I.I.; Ceylan, B.; et al. Predictors of fatality in pandemic influenza A (H1N1) virus infection among adults. BMC Infect. Dis. 2014, 14, 317. [CrossRef]

10. Shi, S.J.; Li, H.; Liu, M.; Liu, Y.M.; Zhou, F.; Liu, B.; Qu, J.X.; Cao, B. Mortality prediction to hospitalized patients with influenza pneumonia: PO2 /FiO2 combined lymphocyte count is the answer. Clin. Respir. J. 2015, 11, 352-360. [CrossRef]

11. Mata-Marín, L.A.; Mata-Marín, J.A.; Vásquez-Mota, V.C.; Arroyo-Anduiza, C.I.; Gaytán-Martínez, J.E.; Manjarrez-Téllez, B.; Ochoa-Carrera, L.A.; Sandoval-Ramírez, J.L. Risk factors associated with mortality in patients infected with influenza A/H1N1 in Mexico. BMC Res. Notes 2015, 8, 432. [CrossRef]

12. Shah, N.S.; Greenberg, J.A.; McNulty, M.C.; Gregg, K.S.; Riddell, J.; Mangino, J.E.; Weber, D.M.; Hebert, C.L.; Marzec, N.S.; Barron, M.A.; et al. Severe Influenza in 33 US Hospitals, 2013-2014: Complications and Risk Factors for Death in 507 Patients. Infect. Control. Hosp. Epidemiol. 2015, 36, 1251-1260. [CrossRef] [PubMed]

13. Huang, W.T.; Chang, C.H.; Hsu, Y.F.; Chuang, J.H. Prognostic factors for mortality in patients hospitalized with influenza complications, in Taiwan. Int. Health 2015, 7, 73-75. [CrossRef] [PubMed]

14. Cvetanovska, M.; Milenkovik, Z.; Uroshevik, V.K.; Demiri, I.; Cvetanovski, V. Factors Associated with Lethal Outcome in Patients with Severe Form of Influenza. Prilozi 2016, 37, 63-72. [CrossRef] [PubMed]

15. Hak, E.; Verheij, T.J.; van Essen, G.A.; Lafeber, A.B.; Grobbee, D.E.; Hoes, A.W. Prognostic factors for influenza-associated hospitalization and death during an epidemic. Epidemiol. Infect. 2001, 126, 261-268. [CrossRef] [PubMed]

16. Gozalo, P.L.; Pop-Vicas, A.; Feng, Z.; Gravenstein, S.; Mor, V. Effect of influenza on functional decline. J. Am. Geriatr. Soc. 2012, 60, 1260-1267. [CrossRef] [PubMed]

17. Garnacho-Montero, J.; Gutiérrez-Pizarraya, A.; Màrquez, J.A.; Zaragoza, R.; Granada, R.; Ruiz-Santana, S.; Rello, J.; Rodríguez, A.; Spanish Society of Intensive Care Medicine and Coronary Units Working Group. Epidemiology, clinical features, and prognosis of elderly adults with severe forms of influenza A (H1N1). J. Am. Geriatr. Soc. 2013, 61, 350-356. [CrossRef] [PubMed]

18. Molbak, K.; Espenhain, L.; Nielsen, J.; Tersago, K.; Bossuyt, N.; Denissov, G.; Baburin, A.; Virtanen, M.; Fouillet, A.; Sideroglou, T.; et al. Excess mortality among the elderly in European countries, December 2014 to February 2015. Eurosurveillance 2015, 20, 21065. [CrossRef]

19. Charlson, M.; Szatrowski, T.P.; Peterson, J.; Gold, J. Validation of a combined comorbidity index. J. Clin. Epidemiol. 1994, 47, 1245-1251. [CrossRef]

20. Katz, S.; Downs, T.D.; Cash, H.R.; Grotz, R.C. Progress in development of the index of ADL. Gerontologist 1970, 10, 20-30. [CrossRef] 
21. Singer, M.; Deutschman, C.S.; Seymour, C.W.; Shankar-Hari, M.; Annane, D.; Bauer, M.; Bellomo, R.; Bernard, G.R.; Chiche, J.D.; Coopersmith, C.M.; et al. The Third International Consensus Definitions for Sepsis and Septic Shock (Sepsis-3). JAMA 2016, 315, 801-810. [CrossRef]

22. Mertz, D.; Kim, T.H.; Johnstone, J.; Lam, P.P.; Science, M.; Kuster, S.P.; Fadel, S.A.; Tran, D.; Fernandez, E.; Bhatnagar, N.; et al. Populations at risk for severe or complicated influenza illness: Systematic review and meta-analysis. BMJ 2013, 347 , f5061. [CrossRef]

23. Bürkle, A.; Moreno-Villanueva, M.; Bernhard, J.; Blasco, M.; Zondag, G.; Hoeijmakers, J.H.J.; Toussaint, O.; Grubeck-Loebenstein, B.; Mocchegiani, E.; Collino, S.; et al. MARK-AGE biomarkers of ageing. Mech. Ageing Dev. 2015, 151, 2-12. [CrossRef] [PubMed]

24. Keef, E.; Zhang, L.A.; Swigon, D.; Urbano, A.; Ermentrout, G.B.; Matuszewski, M.; Toapanta, F.R.; Ross, T.M.; Parker, R.S.; Clermont, G. Discrete Dynamical Modeling of Influenza Virus Infection Suggests Age-Dependent Differences in Immunity. J. Virol. 2017, 91, e00395-17. [CrossRef]

25. Dobson, J.; Whitley, R.J.; Pocock, S.; Monto, A.S. Oseltamivir treatment for influenza in adults: A meta-analysis of randomised controlled trials. Lancet 2015, 385, 1729-1737. [CrossRef]

26. Fiore, A.E.; Fry, A.; Shay, D.; Gubareva, L.; Bresee, J.S.; Uyeki, T.M.; Centers for Disease Control and Prevention (CDC) (2011). Antiviral agents for the treatment and chemoprophylaxis of influenza - recommendations of the Advisory Committee on Immunization Practices (ACIP). MMWR Recomm. Rep. 2011, 60, 1-24.

27. Demicheli, V.; Jefferson, T.; Di Pietrantonj, C.; Ferroni, E.; Thorning, S.; Thomas, R.E.; Rivetti, A. Vaccines for preventing influenza in the elderly. Cochrane Database Syst. Rev. 2018, 2, CD004876. [CrossRef] [PubMed]

28. Beyer, W.E.P.; McElhaney, J.; Smith, D.J.; Monto, A.S.; Nguyen-Van-Tam, J.S.; Osterhaus ADME. Cochrane re-arranged: Support for policies to vaccinate elderly people against influenza. Vaccine 2013, 31, 6030-6033. [CrossRef]

29. Fireman, B.; Lee, J.; Lewis, N.; Bembom, O.; van der Laan, M.; Baxter, R. Influenza vaccination and mortality: Differentiating vaccine effects from bias. Am. J. Epidemiol. 2009, 170, 650-656. [CrossRef] [PubMed] 